\title{
Estrutura e funcionamento familiar e a violência contra idosos
}

\author{
Family structure and functioning and the violence against the elderly
}

Ana Claudia Nunes de Souza Wanderbroocke ${ }^{[a]}$, Carmen Leontina Ojeda Ocampo Morér ${ }^{[b]}$

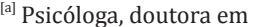
Psicologia pela Universidade Federal de Santa Catarina (UFSC), docente do Programa de Pós-Graduação em Psicologia da Universidade Tuiuti do Paraná (UTP), Curitiba, PR - Brasil, e-mail: anawdb@gmail.com

${ }^{[b]}$ Psicóloga, doutora em Psicologia pela Pontifícia Universidade Católica de São Paulo (PUC-SP), docente do Programa de PósGraduação em Psicologia da Universidade Federal de Santa Catarina (UFSC), Florianópolis, SC - Brasil, e-mail: carmenloom@gmail.com
}

Recebido: 17/08/2011 Received: 08/17/2011

Aprovado: 20/09/2011 Approved: 09/20/2011

\section{Resumo}

0 presente artigo teve como objetivo apresentar fatores relacionados à estrutura e ao funcionamento familiar que contribuem para o aumento da vulnerabilidade do idoso à violência familiar. Os aspectos abordados foram embasados na literatura das áreas da saúde, terapia de família e gerontologia. 0 estudo justifica-se pela perspectiva de aumento contínuo da expectativa de vida no Brasil e no mundo, fato que traz novos desafios para a questão do envelhecimento humano e para as relações intergeracionais. Os dados apresentados servem como subsídio para a reflexão dos profissionais da saúde que assistem famílias e que deparam, em sua práxis, com casos de maus-tratos contra os idosos, para que estejam atentos à sua ocorrência e aos fatores que podem desencadeá-los, de modo a agir preventivamente. 0 estudo procura sensibilizar para a necessidade de pesquisas que privilegiem a participação do idoso vítima de violência familiar, como também que identifiquem nas relações familiares os fatores que protegem os idosos desse agravo.

Palavras-chave: Violência na família. Maus-tratos ao idoso. Relação familiar. Relações intergeracionais.

\section{Abstract}

This article aims to present factors related to family structure and functioning that contribute to increase vulnerability of the elderly to family violence. The discussed issues were based on literature from the fields of health, family therapy and gerontology. The study is justified by the prospect of continued increase in life expectancy in Brazil and worldwide, a fact that brings new challenges to the issue of human aging and intergenerational relations. The data presented serve as an aid to reflection of health professionals who assist families who are faced with cases of abuse against the elderly in their practice, so they can be aware of their occurrence and the factors that can unleash them as a way to act preventively. The study seeks to raise awareness of the need for researches that emphasize the participation of the elderly victims of violence, as well as identify factors that protect them from this injury.

Keywords: Domestic violence. Elder abuse. Family relations. Intergenerational relations.

Psicol. Argum. 2013 jul./set., 31 (74), 395-403 


\section{Introdução}

A violência contra os idosos vem ganhando visibilidade desde a década de 1990 devido ao crescimento populacional de pessoas com mais de 60 anos, bem como das Políticas Públicas promulgadas por diversos países, entre eles o Brasil, a fim de alertar e criar medidas para lidar com o problema.

Para guiar as discussões e ações profissionais, a definição de violência contra idosos proposta pelo International Network for the Prevention of Elder Abuse (Minayo, 2006), é a comumente utilizada: ato único, repetido ou falta de ação que ocorre no contexto de uma relação na qual há uma expectativa de confiança e que causa danos ou perturbações à pessoa idosa. Nesse mesmo sentido, o Plano de Ação para o Enfrentamento da Violência contra a Pessoa Idosa (Brasil, 2005a), usando os termos "maus-tratos" e "abusos" como sinônimos, define sete categorias de violência: física, sexual, emocional ou psicológica, exploração material ou financeira, abandono, negligência e autonegligência.

A violência contra idosos é universal. A literatura de diferentes culturas revela que anciãos de diversos níveis socioculturais e gêneros, etnias, religiões e raças diferentes são vulneráveis (Dong \& Simon, 2010; Gaioli \& Rodrigues, 2008; González \& Zinder, 2009; Wang, 2006). Apesar da certeza de sua ocorrência, levantar a extensão do problema é um grande desafio para pesquisadores do Brasil e do mundo por causa das discrepâncias entre conceituações de violência e metodologias empregadas nos estudos nas estratégias utilizadas para acessar os idosos. Cooper, Selwood e Livingstone (2008), em artigo de revisão sistemática da prevalência de violência contra idosos na população mundial encontraram variação de 3,2\% a 27,5\%; e Espíndola e Blay (2007) localizaram pesquisas indicando coeficiente de abuso físico entre 1,2\% na Holanda, e 18\% na Finlândia. No Brasil, Moraes, Apratto e Reichenheim (2008) levantaram a prevalência de violência física contra o idoso no domicílio, em uma população de baixa-renda assistida pelo Programa de Saúde da Família, em Niterói, com 10,1\% para violência de qualquer forma e 6,3\% para violência grave.

As delegacias de proteção contribuem com dados estatísticos para embasar a compreensão da dimensão dessa problemática. Chaves e Costa (2003), em pesquisa realizada na Delegacia Especializada de Proteção ao Idoso em Belo Horizonte, constataram que $90 \%$ dos casos de maus-tratos e negligência contra pessoas com mais de 60 anos ocorreram nos lares. Na mesma direção, Gaioli e Rodrigues (2008), pesquisando boletins de ocorrência nas delegacias de polícia de Ribeirão Preto, encontraram que $87 \%$ dos idosos foram vítimas de maus-tratos no domicílio.

Se a quantificação ainda não é precisa, os registros efetivados nos serviços de denúncia, delegacias e notificações de profissionais da saúde auxiliam para uma aproximação do perfil da vítima, do agressor e das situações que predispõe os idosos à acentuada vulnerabilidade.

As características dos abusadores delineados na literatura são as seguintes: filhos na meia-idade, homens com maior frequência que mulheres, seguidos por noras, genros e cônjuges que apresentam frequentemente histórico de doenças mentais, desordens de personalidade e abuso de álcool ou drogas; histórico pessoal como vítima de violência familiar ou estrutural; dependência financeira do idoso e moradia muito próxima ou conjunta. As vítimas, na maioria das vezes, são mulheres, com 75 anos ou mais, viúvas, física ou emocionalmente dependentes do agressor, vivem em situação de isolamento social ou em ambiente de pouca comunicação, pouco afeto e é comum apresentarem declínio físico, cognitivo ou funcional (González \& Zinder, 2009; Lowenstein, Eisikovits, Band-Winterstein \& Enosh, 2009; Minayo, 2007).

A violência envolve intensos sentimentos de medo, vergonha e humilhação, principalmente, quando o perpetrador é alguém da própria família, o que pode levar a vítima a silenciar sobre o ocorrido. Entre os motivos associados ao silêncio dos idosos estão o receio de perder: o cuidador, a privacidade (em razão da exposição pública e intervenção exterior), as relações familiares (podendo ser colocado numa instituição). Outros motivos que os levam ao silêncio são o temor de recriminação pelo suposto abusador ou por acharem que ninguém vai acreditar no abuso ou ainda serem indicados como responsáveis pelo comportamento abusivo do outro (Espíndola \& Blay, 2007; Fuster, 2002; González \& Zinder, 2009).

Esses dados revelam o paradoxo que envolve a família. Por um lado, assume grande importância para o bem-estar e desenvolvimento dos seres humanos por ser um lugar de proteção e troca de afeto, espaço de aprendizagem e de formação social, além de ser o espaço natural de referência pessoal (Cerveny \& Berthoud, 2009). Por outro, também é considerada o lugar onde mais ocorrem 
sofrimentos e maus-tratos às crianças, mulheres e idosos (Fuster, 2002). Pode-se pensar também que diante das implicações do envelhecimento para o funcionamento familiar, a vulnerabilidade dos idosos à violência familiar pode aumentar.

Com base no exposto, o presente artigo foi proposto com base no questionamento sobre quais seriam os fatores que dificultam o ajustamento entre as gerações, desencadeando diferentes formas de violência familiar contra o idoso. Dessa forma, o estudo tem como objetivo apresentar fatores relacionados à estrutura e ao funcionamento familiar que podem contribuir para o aumento da vulnerabilidade do idoso à violência familiar, levantados a partir da literatura das áreas da saúde, terapia de família e gerontologia.

\section{Estrutura e funcionamento familiar e a violência contra idosos}

Para Cerveny e Berthoud (1997), as diferentes configurações familiares são moldadas pela correlação das características da estrutura, funcionamento e valores de cada família. As autoras salientam que a estrutura familiar se vislumbra a partir de dados objetivos que desenham o grupo familiar, tais como: número de componentes, sexo, idade, religião, classe socioeconômica, escolaridade, nacionalidade e profissão. Associadas a esses fatores, no processo de desenvolvimento familiar, as posições e os papéis dos integrantes que fazem parte da organização, são desafiados e colocados à prova no jogo interacional e intergeracional, pelo qual se constitui o funcionamento da família. O funcionamento familiar, portanto, é o que configura a dinâmica familiar, relações hierárquicas e de poder.

No que diz respeito às configurações familiares na velhice, a viuvez, que é mais frequente entre as mulheres devido à maior expectativa de vida, interfere consideravelmente no percentual de idosos que vivem sozinhos. Esse fato já foi considerado como abandono familiar e apontado como motivador para o aumento da depressão na velhice. Hoje, considera-se que as famílias, na grande maioria das vezes, não deixam de ser fonte de apoio para os idosos nessa situação. A "intimidade a distância", como se convencionou chamar, apresenta-se como satisfatória tanto para familiares como para idosos, já que preserva fronteiras, garante a independência e a autonomia, permitindo que a reciprocidade de atenção e afeto seja garantida e possibilite ao idoso tempo para a introspecção e revisão de vida, tarefa essencial nessa etapa (Baltes \& Silverberger, 1995; Neri \& Sommerhalder, 2002). Por outro lado, pode-se considerar que quando fatores estressores concorrentes envolvem os familiares, corre-se o risco de negligência em relação às necessidades dos idosos.

Outro arranjo familiar comum na atualidade são os lares multigeracionais, caracterizados por duas, três ou mais gerações em coabitação em razão do declínio físico do idoso, condição financeira insuficiente após aposentadoria, viuvez ou filhos que têm postergado a saída de casa ou antecipado o seu retorno nos casos de desemprego, separação ou problemas de saúde. 0 fato é que gradativamente, um novo fenômeno se evidencia, o não esvaziamento do ninho. Para essas famílias o desafio é a interação entre as gerações, pois a adaptação das pessoas idosas nesses lares exige esforços das partes envolvidas (Camarano \& Ghaouri, 2003; Silveira \& Wagner, 2006).

Carter e McGoldrick (1995) discorreram a respeito dos ajustamentos necessários às famílias durante o processo de envelhecimento de seus membros. A questão principal desse momento, segundo as autoras, é aceitar a mudança dos papéis geracionais, o que envolve que os idosos mantenham o funcionamento e os interesses próprios e/ou de casal em face do declínio fisiológico; lidem com as perdas dos próximos; efetuem revisão e integração de vida; preparem-se para a própria morte; apoiem a atuação mais central da geração intermediária; e que os filhos se abram para a sabedoria e a experiência dos idosos, amparando a geração mais velha sem superfuncionar por ela.

Silva, Alves e Coelho (1997) propõem que o último estágio do ciclo de vida congrega dois aspectos essenciais: o de ruptura e o de continuidade. 0 primeiro associa-se às perdas ou fechamentos característicos do momento, como a aposentadoria, perda de autonomia e a viuvez. Já no segundo aspecto incluem-se os relacionamentos com filhos adultos e netos, ocupação do tempo livre depois da diminuição das demandas funcionais e revisão dos momentos anteriores da vida, que se integram ao presente e balizam as possibilidades futuras. Nesse momento do ciclo de vida, as famílias precisam oferecer um "espaço para envelhecer", social, emocional e geográfico, referido pelos autores como um ambiente de cuidados aos idosos, com ampliação necessária até a morte.

Pensando na integração entre as gerações, Walsh (1995) ressalta o fato de que as famílias num estágio avançado de vida requerem flexibilidade na estrutura, papéis e respostas às novas necessidades

Psicol. Argum. 2013 jul./set., 31 (74), 395-403 
e desafios desenvolvimentais. Segundo a autora, a resposta de cada família às perdas decorrentes do envelhecimento de seus membros depende, em grande parte, da organização das etapas precedentes, lembrando que algumas regras interacionais anteriormente funcionais, podem perder essa qualidade, em determinado momento.

As relações estabelecidas entre pais e filhos são um exemplo da necessidade de flexibilidade e adaptação ao longo do tempo, uma vez que durante a infância e adolescência são fundamentalmente complementares, sustentadas por vínculos de dependência. No entanto, se modificam à medida que as responsabilidades como provedores e cuidadores primários terminam. A autoridade dos pais diminui, seja por ser desnecessária pela independência dos filhos ou pela perda do poder econômico e da saúde. Erbolato (2002) argumenta que, por causa dessas questões, a relação pais-filhos se inverte e é a qualidade dos vínculos anteriores - afeição e sentimentos de dever filial - que assegura a retribuição de suporte e um relacionamento percebido como simétrico. A manutenção de vínculos simétricos é uma das formas de preservar o poder na relação e de evitar os abusos relacionados à objetalização da pessoa idosa.

Nas relações familiares multigeracionais, a convivência pode explicitar diversos interesses e necessidades que se contrapõem e se tornam conflitantes, elevando a tensão intrafamiliar. Lopes e Calderoni (2007) consideram que o envelhecimento dos pais remete à organização interna dos filhos, que confronta com o medo da perda de um ente querido, como também com a possibilidade de ver nos pais idosos um espelho do próprio futuro. Além disso, normalmente, os filhos estão na meia-idade, fase em que os adultos encontram-se sobrecarregados com responsabilidades em relação a trabalho, filhos e lidando com o seu processo de envelhecimento. Essa colocação vai ao encontro do que foi proposto por Walsh (1995) como interação geracional cruzada, isto é, quando as tarefas do estágio tardio da vida dos idosos interagem com as questões de vida das gerações mais jovens, aumentando a probabilidade de ocorrerem maus-tratos.

Entre as relações intergeracionais, a experiência de se tornar avô é apontada como oportunidade de reviver questões relacionadas à criação dos filhos e retomar os vínculos de dependência que coloca os idosos em posição de destaque. Quanto melhor a condição física dos avôs, maior a importância desse papel nas famílias. Entretanto, se por um lado o neto é a possibilidade de se imortalizar, também pode representar a concretização da passagem do tempo, trazendo sentimentos ambivalentes ao idoso pelo confronto com a finitude (Erbolato, 2002; Lopes \& Calderoni, 2007). Avós superenvolvidos podem suscitar, ainda, problemas na dinâmica familiar e provocar conflitos conjugais dos filhos ou em triangulações com netos, como ocorre quando os avós não aceitam a passagem do tempo e desejam dar continuidade ao papel materno/paterno competindo ou impedindo o filho de exercê-lo (Falcão, Dias, Bucher-Maluschke \& Salomão, 2006; Walsh, 1995).

O conflito entre as gerações é foco de vários estudos na atualidade e uma das questões frequentemente mencionadas nas pesquisas sobre violência familiar. A ambivalência é um sentimento normalmente presente nos familiares, os quais ficam entre o desejo de cuidar devido à afeição à pessoa idosa e as restrições que a sua presença pode significar, incluindo condições de moradia e falta de privacidade. 0 idoso, por sua vez, pode sentir-se um peso para a família, desenvolvendo sentimento de culpa e desgosto por sua situação de dependência, o que pode gerar raiva e frustração para si e para o ambiente (Soliz \& Harwood, 2003; Zhang, 2004).

Silva et al. (1997) citam que uma das tarefas do estágio tardio das famílias é oferecer um "espaço para envelhecer" e, a partir disso, refletem que na atual conjuntura a grande maioria das famílias é urbana, nuclear, com poucos filhos e se constitui numa célula autoprodutiva porque produz de forma especializada, necessitando cada vez de mais horas de trabalho. Ressaltam que se trata de um modelo quase antropofágico, pois quanto mais produzem, mais necessitam, mas nem sempre alcançam com esse esforço qualidade de vida, e vivem em espaços cada vez mais reduzidos e isoladas. Consideram as mudanças estruturais e as de funcionamento das famílias como fatores potenciais para a ocorrência de violências, justificando que a disponibilidade para oferecer um espaço para o envelhecimento de seus membros nem sempre está presente. Diante dessas transformações, os idosos deixam de ocupar um lugar de respeito para passar a uma quase invisibilidade nas famílias. Ainda, suas necessidades e direitos não são reconhecidos; portanto, são coisificados e tornam-se potenciais vítimas de violência, identificados como empecilho para a realização dos mais jovens.

Mudanças na estrutura e funcionamento das famílias também são apontadas por Mattos (2009) 
como fatores que aumentam a vulnerabilidade dos idosos. Eles salientam que o espaço de cuidados oferecidos aos mais velhos relaciona-se à diminuição do número de filhos, a mudanças no mundo do trabalho, que levam à maior mobilização e acesso à tecnologia e informação, bem como à reorganização da família pós-divórcio, recasamento, entre outros. Além disso, na opinião da autora, se o idoso pertenceu a uma grande família de origem, mais suportiva e acolhedora, frequentemente sente-se excluído e desamparado, pois não teve a experiência dos modelos de família da atualidade.

Em uma sociedade capitalista a situação econômica do idoso lhe confere situação paradoxal. Por um lado, uma boa condição financeira é tomada como sinal de competência, sabedoria e poder, pois, quem é mais independente tem a probabilidade de ser considerado positivamente. Nesse sentido, é interessante notar que, de acordo com o Instituto Brasileiro de Geografia e Estatística (IBGE) (2007), os idosos ocupam cada vez mais a posição de chefia econômica dos domicílios quando moram com os familiares e o mais comum é morarem com os filhos, o que contribui para que mantenham um lugar de poder e respeito nas relações familiares.

Por outro lado, as questões financeiras são um problema enfrentado hoje por diversas famílias, seja pela dependência do idoso em relação à família ou da família em relação ao idoso, podendo culminar em abusos financeiros. Uma variante do abuso financeiro é a apropriação indevida de patrimônio através da violência, que Menezes (1999), citado por Santos, Silva, Carvalho e Menezes (2007), refere como uma das queixas mais registradas nas Delegacias de Polícia especializadas para idosos. Segundo a autora, entre os idosos isso se torna comum porque eles permitem que os agressores se apropriem de seus bens por temerem a solidão, a vida em asilos e clínicas geriátricas. Os agressores, depois da apropriação dos bens, detêm o poder sobre o idoso e seguem coagindo, ameaçando e perpetrando o ciclo da violência.

Chaves e Costa (2003) também descrevem como as famílias agem na tentativa de forçar procurações para acesso aos bens dos mais velhos. Nesse quadro se incluem a apropriação ou venda sem consentimento, expulsão do idoso do imóvel ou seu confinamento em espaços minúsculos. 0 estudo de Setterlund, Cheryl, Wilson e Rosenman (2007) permite refletir a respeito do abuso financeiro, ao indicar que ele ocorre mais por oportunismo ou por ganância de quem tem vínculo com o idoso ou administra suas finanças do que por necessidades financeiras, estresse ou sobrecarga, consequentes da relação. Nessas situações, dizem os autores, é difícil diferenciar comportamentos protetivos ou abusivos por aquele que cuida das finanças do idoso, além do que, essa pessoa costuma impor o seu poder, mesmo que indiretamente. Discutem ainda até que ponto o abuso não se dá pelo preconceito, quando se pensa que o idoso já não precisa dispor de seus recursos ou patrimônio.

Outro aspecto a se considerar como potencializador dos conflitos intergeracionais é quando os idosos necessitam de cuidados, pela fragilização decorrente do avançar dos anos ou pelo surgimento de alguma doença, gerando dificuldades para os netos e filhos. Os filhos precisam assumir cada vez mais a função antes exercida pelos pais; e além do aumento de tarefas instrumentais, sentimentos profundos estão envolvidos nessa situação. Neri e Sommerhalder (2002) advertem que o processo de cuidar também não é homogêneo ou linear: "Numerosos elementos afetivos e cognitivos mesclam-se com tarefas e papéis, criando uma verdadeira história do cuidado, que alterna preparação, aquisição e consolidação do papel, afastamento e luto" (Neri \& Sommerhalder, 2002, p. 50).

0 aspecto apresentado pelas autoras é relevante e induz a uma análise já que o estresse gerado no cuidador pela dependência crescente dos idosos é apontado como fator de risco para a violência, sendo os cuidadores principais os potenciais agressores (Cooper, Blanchard, Selwood, Walker \& Livingston, 2010; Perez-Rojo, Izal, Montorio \& Penhale, 2009; Wang, Lin, Tseng \& Chang, 2009). Nesse sentido, estudos como o de Moraes et al. (2008) e de González e Zinder (2009) indicam maior índice de ocorrência de violência física entre os idosos que apresentam mais patologias, perda de autonomia física ou cognitiva e que demandam mais cuidados.

Neri e Sommerhalder (2002) revisaram a bibliografia nacional e internacional sobre o impacto do cuidar sobre o bem-estar do cuidador familiar de idosos. Os autores ressaltam que a maioria das pesquisas se direciona a cuidadores de idosos portadores de demências, os quais exigem muita atenção justamente porque apresentam perdas das capacidades físicas e cognitivas crescentes e irreversíveis. Entre os dados encontrados estão os efeitos negativos sobre a saúde física e mental do cuidador,

Psicol. Argum. 2013 jul./set., 31 (74), 395-403 
refletidos em prevalência de doenças psiquiátricas, utilização maior do que o usual de drogas psicotrópicas, mais doenças somáticas, pior saúde percebida, isolamento social, estresse pessoal e familiar, e sentimentos de ter que cumprir uma obrigação pesada e cheia de tensão.

Porém, Minayo (2007) alerta que pesquisas recentes desmistificam a ideia de que o estresse do cuidador é um fator de risco para a violência e a atuação dos cuidadores principais como prováveis agressores; ele defende que, apesar de o estresse do cuidador contribuir para a existência de maus-tratos, ele não é determinante. 0 autor explica essa afirmação apontando que a qualidade da relação prévia entre cuidador e idoso parece ser um indicador confiável e assinala que a falta de vínculos significativos ou "vínculos frouxos" desponta como causa mais frequente. Dessa forma, dar visibilidade às relações familiares envolvendo cuidados à pessoa idosa é um provável caminho para evitar situações de violência e maus-tratos.

Pasinato, Camarano e Machado (2006) ao se referirem à problemática dos cuidadores, destacam a carência de programas governamentais direcionados ao amparo dos idosos que se tornam dependentes. Por esse motivo, a família assume a responsabilidade pelos cuidados apesar do deficit de informação e de capacitação para essas atividades. Diante desse panorama, as famílias que cuidam ficam suscetíveis à pressão financeira, sobrecarga física e aumento do isolamento social do cuidador. Por isso, os pesquisadores consideram urgente a implantação de serviços e programas que gerem suporte à família brasileira para cuidar dos idosos em seus lares. Entre os exemplos indicam a criação de instituições intermediárias de cuidado, como centros-dia ou programas intergeracionais como possíveis alternativas para conter a violência dentro da família e diminuir os índices de negligência e abandono.

A personalidade do idoso e a postura por ele assumida fazem diferença na sua integração à rotina e funcionamento da família. É possível observar diferenças quanto ao gênero, já que a mulher comumente toma uma atitude mais pró-ativa quanto às questões de saúde pessoal, está mais disponível aos cuidados de familiares dependentes e busca participação social. Por outro lado, os homens parecem ter maior dificuldade em deixar a vida produtiva após a aposentadoria, entristecem-se com mais frequência e apresentam maior dificuldade em adaptar-se a essa fase (Mattos, 2009).

0 fato de os idosos se transformarem em vítimas na velhice também se relaciona à violência ou abandono de familiares, por eles perpetrados no passado, além de comportamentos disruptivos, agressividade e atitudes provocativas em relação aos familiares. A transmissão transgeracional da violência é um assunto vastamente estudado na área de terapia familiar (Antoni, Barone \& Koller, 2006; Fuster, 2002; Ravazzola, 2005; Silva, Falbo Neto \& Cabral Filho, 2009). Ela é também apontada como fator gerador da violência contra idosos (Faleiros \& Brito, 2009; Penso \& Marais, 2009; Santos et al., 2007).

Para analisar os motivos que levam um familiar a agir com violência em relação a um idoso, há que se levar em conta não só características dos idosos ou da família, já que se trata de um fenômeno multideterminado e que deve ser analisado em sua complexidade. A conjuntura econômica e política do país afeta as famílias que, por sua vez, refletem esses aspectos na relação com os idosos. É o que ocorre quando as pessoas não fixam residência, habitam em locais com alta densidade populacional ou com características não homogêneas, dificultando a coesão de seus integrantes e gerando o isolamento social. Outro exemplo são as comunidades com problemas de tráfico de drogas e alto índice de desemprego, já que áreas de pobreza, com deterioração física e com pouco suporte institucional, são mais propensas à violência (Brasil, 2005b).

Apesar de a violência familiar se manifestar em todas as classes sociais, estudiosos da área de família demonstram a relação entre os ambientes de pobreza e a significativa incidência de maus-tratos (Narvaz \& Koller, 2006; Parente, Nascimento \& Vieira, 2009). No caso dos idosos, as vulnerabilidades podem se somar às limitações impostas pela idade, saúde debilitada, dependência, além da pobreza que expõe as famílias a diversas formas de violência durante seu ciclo vital (Penso \& Morais, 2009).

Quanto às consequências da violência familiar nos idosos, elas se assemelham às implicações para as pessoas das demais idades; no entanto, potencializadas quando há maior fragilidade orgânica. Pesquisas sobre o tema registram aumento de comorbidades, quadros de depressão e de estresse pós-traumático, sentimentos de desesperança, alienação, culpa, 
vergonha, medo, ansiedade, negação e o aumento de estresse nas relações interpessoais, aumentando o risco de morte como decorrência da violência familiar na velhice (Moraes et al., 2008; González \& Zinder, 2009). Entretanto, os dados oferecidos pela literatura a esse respeito ainda são limitados.

\section{Consideracões Finais}

Este estudo percorreu diferentes aspectos que elevam a vulnerabilidade do idoso à violência, considerando a estrutura e o funcionamento das famílias, bem como características pessoais do idoso e fatores externos à família que influenciam sua dinâmica. Em função da perspectiva de contínuo aumento populacional de idosos, cabe aos profissionais que assistem famílias estarem atentos à ocorrência de possíveis maus-tratos, bem como aos fatores que podem desencadeá-los a fim de agir preventivamente.

Como a violência contra os idosos só se tornou foco de estudos nas últimas décadas, os dados de pesquisa ainda não revelam todos os aspectos envolvidos no problema. Inclusive, a revisão realizada por Minayo, Souza e Paula (2010) evidenciou o quanto ainda é irrelevante o número de trabalhos tendo o idoso como interlocutor. Uma explicação plausível para justificar a significativa ausência da voz da pessoa idosa nas pesquisas é porque os estudos sobre esse assunto têm como objeto principal o idoso fragilizado física, e/ou cognitiva ou funcionalmente dependente. Ainda, existe o fato de os serviços de denúncia e acompanhamento de vítimas estarem em processo de estruturação no Brasil, dificultando o acesso a essa população, que nem sempre apresenta condições de participar de pesquisas.

Sugere-se que pesquisadores interessados nessa área invistam em estudos que privilegiem a participação do idoso, como também, em estudos que identifiquem nas relações familiares os fatores que os protegem da vivência de maus-tratos.

\section{Referências}

Antoni, C., Barone, L. R., \& Koller, S. H. (2006). Violência e pobreza: Um estudo sobre vulnerabilidade e resiliência familiar. In D. Dell'Aglio, S. H. Koller \& M. A. Yunes (Org.). Resiliência e psicologia positiva: Interfaces do risco à proteção (pp. 141-172). São Paulo: Casa do Psicólogo.
Baltes, M. M., \& Silverberger, G. S. (1995). A dinâmica dependência autonomia no curso de vida. In A. L. Néri (Org.). Psicologia do envelhecimento (pp. 73-110). Campinas: Papirus.

Brasil (2005a). Presidência da República. Plano de ação para o enfrentamento da violência contra a pessoa idosa. Brasília: Subsecretaria de Direitos Humanos.

Brasil (2005b). Ministério da Saúde. Impacto da violência na saúde dos brasileiros. Brasília: Ministério da Saúde.

Camarano, A. A., \& Ghaouri, S. K. (2003). Famílias com idosos: ninhos vazios? Instituto de Pesquisa Econômica Aplicada, IPEA, Rio de Janeiro. Recuperado em 5 set. 2013, de http://www.ipea.gov.br/agencia/images/ stories/PDFs/TDs/td_0950.pdf

Carter, B., \& McGoldrick, M. (1995). As mudanças no ciclo de vida familiar. Uma estrutura para a terapia familiar. In B. Carter \& M. McGoldrick. As mudanças no ciclo de vida familiar. Uma estrutura para a terapia familiar. (2. ed., pp. 7-39). Porto Alegre: Artes Médicas.

Cerveny, C. M. O., \& Berthoud, C. M. E. (1997). Família e ciclo vital: Nossa realidade em pesquisa. São Paulo: Casa do Psicólogo.

Cerveny, C. M. O., \& Berthoud, C. M. E. (2009). Ciclo vital da família brasileira. In L. C. Osório, \& M. E. P. Valle (Org.). Manual de terapia familiar. (pp. 25-37). Porto Alegre: Artmed.

Chaves, P. G. S., \& Costa, P. L. (2003). A violência afetiva e a violência doméstica contra os idosos. Recuperado em 3 maio 2008, de www.mj.gov.br/Senasp/senasp/ artigo/violen_idoso.html

Cooper, C., Blanchard, M., Selwood, A., Walker, Z., \& Livingston, G. (2010). Family cares distress and abusive behavior: Longitudinal study. The British Journal of Psychiatry, 196(6), 480-485.

Cooper, C., Selwood, A., \& Livingstone, G. (2008). The prevalence of elder abuse and neglect: A systematic review. Age and Aging, 37, 151-160.

Dong, X., Beck, T., \& Simon, M. A. (2010). The association of gender, depression and elder mistreatment in a community-dwelling Chinese population: The modifying effect of social support. Archives of Gerontology and Geriatrics, 50(2), 202-208.

Erbolato, R. M. P. L. (2002). Relações sociais na velhice. In E. V. Freitas, L. Py, A. L. Néri, F. A. X. Cançado, M. L. Gorzoni \& S. M. Rocha. Tratado de geriatria e gerontologia. Rio de Janeiro: Guanabara Koogan. 
Espíndola, C. R., \& Blay, S. (2007). Prevalência de maus-tratos na terceira idade: Revisão sistemática. Revista de Saúde Pública, 41(2), 301-306.

Falcão, D. V. S., Dias, C. M. S. B., Bucher-Maluschke, J. S. N. F., \& Salomão, N. M. R. (2006). As relações familiares entre as gerações: possibilidades e desafios. In D. V. S. Falcão \& C. M. S. B. Dias (Org.). Maturidade e velhice: Pesquisas e intervenções psicológicas. (Vol.1, pp. 5980). São Paulo: Casa do Psicólogo.

Faleiros, V. P., \& Brito, D. O. (2009). Representações da violência intrafamiliar por idosos e idosas. In V. P. Faleiros, A. M. L. Loureiro \& M. A. Penso (Org.). O conluio do silêncio - A violência intrafamiliar contra a pessoa idosa. (pp. 1-20). São Paulo: Roca.

Fuster, E. G. (2002). Las víctimas invisibles de la violencia familiar. El extraño iceberg de la violencia doméstica. Buenos Aires: Paidós.

Gaioli, C. C. L. O., \& Rodrigues, R. A. P. (2008). Occurrence of domestic elder abuse. Revista Latino-Americana de Enfermagem, 16(3), 465-70.

González, M. G. R., \& Zinder, N. S. (2009). Factores asociados con el auto-reporte de maltrato en adultos mayores de México. Revista Chilena de Salud Pública, 13(2), 90-99.

Instituto Brasileiro de Geografia e Estatística, [IBGE]. (2007). IBGE divulga indicadores sociais dos últimos dez anos. Recuperado em 30 jun. 2008, de http://saladeimprensa.ibge.gov.br/noticias?view=noticia\&id $=1 \&$ busca $=1$ \&idnoticia $=987$

Lopes, R. G. C., \& Calderoni, S. Z. (2007). 0 idoso na família: expansão de possibilidades ou retração? In M. Papaléu Netto (Org.). Tratado de gerontologia (2. ed. rev. e ampl., pp. 225-231). São Paulo: Atheneu.

Lowenstein, A., Eisikovits, Z., Band-Winterstein, T., \& Enosh, G. (2009). Is elder abuse and neglect a social phenomenon? Data from the First National Prevalence Survey in Israel. Journal of Elder Abuse and Neglect, 21(3), 253-277.

Mattos, E. T. B. (2009). Família com idosos. In L. C. Osório \& M. E. P. Valle (Org.). Manual de terapia familiar. (pp. 312-322). Porto Alegre: Artmed.

Minayo, M. C. S. (2006). Violência e saúde. Rio de Janeiro: Editora Fiocruz.

Minayo, M. C. S. (2007). Violência contra a pessoa idosa: $\mathrm{O}$ direito pelo avesso. In M. Papaléu Netto (Org.). Tratado de gerontologia. (2. ed. rev. e ampl., pp. 199210). São Paulo: Atheneu.
Minayo, M. C. S., Souza, E. R., \& Paula, D. R. (2010). Revisão sistemática da produção acadêmica brasileira sobre causas externas e violências contra a pessoa idosa. Ciência e Saúde Coletiva, 15(8), 2719- 2728.

Moraes, C. L., Apratto Jr., P.C., \& Reichenheim, M. E. (2008). Rompendo o silêncio e suas barreiras: Um inquérito domiciliar sobre a violência doméstica contra idosos em área de abrangência do Programa Médico de Família de Niterói, Rio de Janeiro, Brasil. Cadernos de Saúde Pública, 24(10), 2289-2300.

Narvaz, M. G., \& Koller, S. H. (2006). Mulheres vítimas de violência doméstica: Compreendendo subjetividades assujeitadas. Psico, 31(1), 7-13.

Neri, A. L. \& Sommerhalder, C. (2002). As várias faces do cuidado e do bem-estar do cuidador. In A. L. Néri (Org.). Cuidar de idosos no contexto da família: Questões psicológicas e sociais. Campinas: Alínea.

Parente, E. O., Nascimento, R. O., \& Vieira, L. J. E. S. (2009). Enfrentamento da violência doméstica por um grupo de mulheres após a denúncia. Revista Estudos Feministas, 17(2), 445-465. Recuperado em 10 set. 2013, de http:// www.scielo.br/pdf/ref/v17n2/08.pdf

Pasinato, M. T., Camarano, A. A., \& Machado, L. (2006). Idosos vítimas de maus tratos domésticos: Estudo exploratório das informações dos serviços de denúncia. Instituto de Pesquisa Econômica Aplicada, IPEA, Rio de Janeiro. Recuperado em 5 set. 2013, de http://www.ipea.gov.br/ portal/index.php?option=com_content\&view= article\&id $=4360$

Penso, M. A., \& Morais, I. A. (2009). 0 ciclo da violência em famílias com idosos. In V. P. Faleiros, A. M. L Loureiro \& M. A. Penso (Org.). O conluio do silêncio - A violência intrafamiliar contra a pessoa idosa. (pp. 47-62). São Paulo: Roca.

Perez-Rojo, G., Izal, M., Montorio, I., \& Penhale, B. (2009). Risk factors of elder abuse in a community dwelling Spanish sample. Archives of Gerontology and Geriatrics, 49(1), 17-21.

Ravazzola, M. C. (2005). Histórias infames: Los malostratos en las relaciones. Buenos Aires: Paidós.

Santos, A. C. P., Silva, C. A., Carvalho, L. S., \& Menezes, M. R. (2007). A construção da violência contra idosos. Revista Brasileira de Geriatria e Gerontologia, 10(1): 129-140. 
Setterlund, D., Cheryl, T., Wilson, A. M., \& Rosenman, L. (2007). Understanding financial elder abuse in families: The potential of routine activities theory. Ageing \& Society, 27(4), 599-614.

Silva, J. L., Alves, L. F., \& Coelho, M. R. M. (1997). A família em fase última. In C. M. O. Cerveny \& C. M. E Berthoud. Família e ciclo vital: nossa realidade em pesquisa. (pp. 121-132). São Paulo: Casa do Psicólogo.

Silva, M. A., Falbo, G. H., Neto, \& Cabral, M. T., Filho. (2009). Maus-tratos na infância de mulheres vítimas de violência. Psicologia em Estudo, 14(1), 121-127.

Silveira, P. G., \& Wagner, A. (2006). Ninho cheio: A permanência do jovem em sua família de origem. Estudos de Psicologia, 23(4), 441-453.

Soliz, J. \& Harwood, J. (2003). Perceptions of communication in a family relationship and the reduction of intergroup prejudice. Journal of Applied Communication Research, 31(4), 320-345.
Walsh, F. (1995). A família no estágio tardio da vida. In B. Carter \& M. McGoldrick. As mudanças no ciclo de vida familiar: Uma estrutura para a terapia familiar. Porto Alegre: Artes Médicas.

Wang, J. J. (2006). Psychological abuse and its characteristic correlates among elderly Taiwanese. Achieves of Gerontology and Geriatrics, 42(3), 307-318.

Wang, J. J., Lin, M. F., Tseng, H. F., \& Chang, W. Y. (2009). Caregiver factors contributing to psychological elder abuse behavior in long-term care facilities: A structural equation model approach. International Psychogeriatrics, 21(2), 314-320.

Zhang, Y. B. (2004). Initiating factors of Chinese intergenerational conflict: Young adults' written accounts. Journal of Cross Cultural Gerontology, 19(4), 299-319. 